\title{
Synchrotron FTIR analysis of drug treated ovarian A2780 cells: an ability to differentiate cell response to different drugs? $\uparrow+$
}

\author{
Kevin R. Flower, ${ }^{* a}$ Intisar Khalifa, ${ }^{b}$ Paul Bassan, ${ }^{b}$ Damien Démoulin, ${ }^{b}$ Edward Jackson, ${ }^{b}$ Nicholas P. Lockyer, ${ }^{b}$ \\ Alan T. McGown, ${ }^{c}$ Philip Miles, $^{a}{\text { Lisa } \text { Vaccari }^{d}{ }^{\text {and }} \text { Peter Gardner }}^{b}$
}

\author{
Received 23rd July 2010, Accepted 2nd November 2010 \\ DOI: 10.1039/c0an00564a
}

Recently a new di-gold(I) organometallic complex [1,3-( $\left.\left.\mathrm{Ph}_{3} \mathrm{PAu}\right)_{2}-\mathrm{C}_{6} \mathrm{H}_{4}\right](\mathrm{KF} 0101)$ has been synthesised and found to exhibit cytotoxic activity in vitro. Subsequently it has been demonstrated that KF0101 shows little or no cross-resistance against a number of the cisplatin resistant ovarian cancer cell lines in vitro suggesting a different mode of action for the drug. In this study, syncrotron radiation infrared microspectroscopy (SR-IRMS) has been used on drug treated single A2780 cells in order to determine if this different mode of action can be identified spectroscopically. The aim of the study was to establish: (i) if single cell SR-IRMS could be used to give insight into the cellular response on treatment with different cytotoxic agents relative to non-treated cells (control) and (ii) that if the cytotoxic drugs elicit a different biochemical response these responses could be distinguished from each other. The most striking features obtained after Principal Componants Analysis (PCA) of Resonant Mie Scattering (RMieS) corrected single cell spectra of drug treated ovarian A2780 cells are: (i) The spectra obtained for the control are quite heterogeneous and several hundred spectra are required to adequately define the nature of the control; (ii) after drug treatment at the IC50 level for $24 \mathrm{~h}$ with cisplatin, KF0101, methotrexate, paclitaxel or 5-fluorouracil the cell spectra, as represented on a PCA scores plot, generally concentrate in certain well defined areas of the control, there are however a small number of spectra that fall outside of the area defined by the control; and (iii) a differentiation between cell spectra obtained on treatment with different drugs is observed which fits well with different in vitro cell culture behaviour and a flow cytometry cell cycle analysis of the contol and drug treated cells. Inspection of the loading plots shows that $\mathrm{PC} 1$ is essentially the same for all plots and reflects changes in cell biochemistry related to the cell cycle. PC2, however, on comparison of the control versus cisplatin or cisplatin versus KF0101 is indicative of differences induced by drug treatment and has been termed as cell cycle-plus behaviour. These data are shown to be consistent with that obtained using bench-top IRMS by averaging a number of single cell spectra and carrying out a PCA, but SR-IRMS offers more insight into how the drug is affecting the cell population. More importantly, this approach enables the influence of the cell cycle on both the control and drug treated samples to be taken into consideration when evaluating the drug-cell interaction.

\footnotetext{
${ }^{a}$ School of Chemistry, University of Manchester, Manchester, M13 9PL, UK.E-mail:kevsnuts@hotmail.com

${ }^{b}$ Manchester Interdisciplinary Biocentre, The University of Manchester, Manchester, M1 7DN, UK

'School of Environmental and Life Sciences, University of Salford, Salford, M5 4WT, UK

${ }^{d}$ Elettra Synchrotron Light Laboratory, SS14 Km 163.5, 34149 Basovizza, Trieste, Italy

$\uparrow$ This paper was submitted as part of an Analyst themed issue on Optical Diagnosis. The issue includes work which was presented at SPEC 2010 Shedding Light on Disease: Optical Diagnosis for the New Millennium, which was held in Manchester, UK June 26th-July 1st 2010. Other papers on this topic can be found in issue 12 of vol. 135 (2010). This issue can be found from the Analyst homepage (http://www.rsc.org/analyst).

\$ Electronic supplementary information (ESI) available: Spectral correction output after 1 iteration and 8 iterations of the RMieS correction programme; individual scatter plots for the 30 cells in each collection allocation of the control; comparison of cells treated with two different drugs; comparison of cells treated with three different drugs; comparison of cells treated with all drugs and the control. See DOI: $10.1039 / \mathrm{c} 0 \mathrm{an} 00564 \mathrm{a}$
}

\section{Introduction}

The application of vibrational spectroscopy aimed at detecting disease in human cells started in the early $1990 \mathrm{~s}^{1}$ but was hampered by the fact that the data was collected over an area of several square millimetres and the samples being studied contained inherent heterogeneity. With the development of both infrared and Raman micro-spectroscopic methods, where data are collected through a microscope, high quality images with high spectral and spatial resolution can be acquired. This gives the necessary methodology for correlation between disease and spectra to be attained. ${ }^{2}$ A detailed account of these early developments is available, ${ }^{3}$ as are a number of more recent reviews. ${ }^{2,4,5}$

SR-IRMS has been applied to the study of single cells. ${ }^{6-16} \mathrm{~A}$ major limitation to the application of this technique is infrared spectra from single cells often exhibit highly distorted baselines, and derivative-like peak shapes of the strongest bands such as the amide I. ${ }^{17-19}$ These distortions result in changes in apparent peak intensity and position rendering a detailed interpretation of biochemical changes in the cells unreliable. Consequently much 
effort has been put into understanding the origin of this distortion $^{20-22}$ and it is now known that the predominant cause is Resonant Mie Scattering (RMieS). ${ }^{23}$ Recently a correction algorithm has been developed that enables spectra to be corrected for RMieS thus removing scattering effects and leaving the absorption spectrum of the sample. ${ }^{24}$ Separating scattering contributions from biochemical changes in spectra is particularly important in the case of drug treated cells since, in addition to a biochemical response, cytotoxic agents often change the morphology of cells; for example, rounding of the cells which results in a change in the scattering properties. The development of the RMieS correction enables the effect of these changes in cell morphology to be separated from the changes in biochemistry paving the way for a biological interpretation of drug treated single cell spectra. ${ }^{25}$

Despite the problems caused by scattering several attempts have been made to study drug-cell interactions using FTIR. In a recent report ${ }^{26}$ by Goormaghtigh et al. the authors have shown that prostate cancer PC3 cells, a line that has been extensively studied by FTIR spectroscopy, ${ }^{12,27-32}$ when treated with ouabain, a new potential antitumour drug, show signatures of activity relating to a modification of cell lipids. Draux et al. ${ }^{8}$ used both bench top and SR-IRMS to study sub-lethal doses of Gemcitabine on the non-small cell lung cancer cell line Calu-1 and were able to detect significant spectral differences between groups of cells where cell proliferation was weakly, moderately and highly inhibited. Importantly some of the changes observed in the spectra $e . g$. increase in RNA bands were attributed to changes in the cell cycle since Gemcitabine is known to cause cell accumulation in the G1 phase. The SR-FTIR spectra, however, were the average of 30 cells and thus information regarding the cell cycle at the single cell level will have been lost. Other work relating directly to the cell cycle has been described using both SRIRMS $^{8,15,16}$ and bench-top IRMS. ${ }^{33-35}$ Significant changes in spectra profile have been observed for each of the phases and these must be taken into account in the analysis of drug treated cells where the distribution of cells in each phase can change dramatically.

Recently a new di-gold(I) organometallic complex [1,3$\left.\left(\mathrm{Ph}_{3} \mathrm{PAu}\right)_{2}-\mathrm{C}_{6} \mathrm{H}_{4}\right]^{36}$ has been prepared Fig. 1, and found to show little or no cross-resistance against cisplatin resistant cells in vitro, indicating the complex acts either by a different mechanism or at a different point in the cell cycle.

In this study SR-FTIR has been used to investigate the interaction of KF0101 and cisplatin with A2780 cells. For comparison methotrexate, paclitaxel and 5-fluorouracil which operate via different modes of action have also been investigated. ${ }^{37-41}$ Specifically SR-IRMS has been used to see if: (i) Drug treated A2780 cells could be distinguished from non-drug treated cells (control); (ii) Drugs that operate by different mechanisms elicit a different cellular response and can be distinguished from each other; (iii) The spectral response observed is simply due to the cell

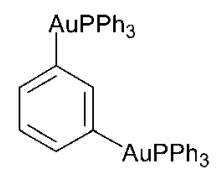

Fig. 1 Molecular structure of $\left[1,3-\left(\mathrm{Ph}_{3} \mathrm{PAu}\right)_{2}-\mathrm{C}_{6} \mathrm{H}_{4}\right](\mathrm{KF} 0101)$. cycle; and (iv) Data is consistent across different synchrotron sources and with that obtained using bench-top IRMS.

\section{Experimental}

\subsection{Sample preparation}

Cisplatin, methotrexate, paclitaxel and 5-fluorouracil were purchased from commercial sources and used without further purification; 1,3- $\left(\mathrm{Ph}_{3} \mathrm{PAu}\right)-\mathrm{C}_{6} \mathrm{H}_{4}$ was prepared in an analogous manner previously described for $1,4-\left(\mathrm{Ph}_{3} \mathrm{PAu}\right)-\mathrm{C}_{6} \mathrm{H}_{4}{ }^{36}$

A2780 cells were seeded and grown directly on MirrIR slides in six-well plates. When cell coverage had reached $70 \%$ coverage they were drug treated with cisplatin $(500 \mathrm{nM}), \mathrm{KF} 0101(50 \mathrm{nM})$, methotrexate $(20 \mu \mathrm{M}), 5 \mathrm{FU}(1000 \mathrm{nM})$ and paclitaxel $(2000 \mathrm{nM})$ (IC50) for $24 \mathrm{~h}$. The cells were then fixed in formalin and washed several times with PBS and air dried as previously described. ${ }^{13}$ Each experiment was carried out in triplicate.

\subsection{Flow cytometry}

A2780 cells were grown in humidified incubator with $5 \% \mathrm{CO}_{2}$ at $37{ }^{\circ} \mathrm{C}$ in RPMI-1640 medium supplemented with $1 \%$ L-Glutamine and $10 \%$ FCS. For cell cycle analysis cells, in exponential growth, were treated under the same conditions as for the SRIRMS (cisplatin (500 nM), KF0101 (50 nM), methotrexate (20 $\mu \mathrm{M})$, 5FU (1000 nM), paclitaxel (2000 nM) for $24 \mathrm{~h})$. The cells were then detached by trypsinisation and fixed in ethanol $(70 \%)$. Cells were treated with RNAse (Sigma, $1 \mathrm{mg} \mathrm{ml}^{-1}, 30 \mathrm{~min}$, $37{ }^{\circ} \mathrm{C}$ ) and re-suspended in propidium iodide solution (Sigma, $50 \mu \mathrm{g} \mathrm{ml}^{-1}$ in PBS). Analysis of DNA content was carried out on a Partec Cyflow cytometer using the Partec cell cycle analysis software.

\subsection{FTIR measurements}

Infrared spectra of individual cells were obtained at two different synchrotron facilities. At the SRS, Daresbury laboratory, infrared spectra were recorded on Station 11.1 12,43 where synchrotron light is directed into a Nicolet Nexus FTIR spec-

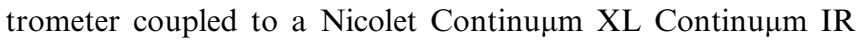
microscope fitted with $32 \times$ objective, mapping stage, and 50 micron MCT detector. Individual cells were selected using the microscope and the aperture adjusted to the cell size (generally $15 \times 15 \mu \mathrm{m}) .30$ spectra were recorded in turn from each replicate. The spectra were acquired over 256 scans and $4 \mathrm{~cm}^{-1}$ resolution. At the Elettra Synchrotron Laboratory, Trieste, Italy the data were collected using the infrared SISSI (Synchrotron Infrared Source for Spectroscopy and Imaging) beamline. ${ }^{44}$ FTIR transflection spectra were collected using a Bruker Hyperion 3000 Vis-IR microscope equipped with a mid-band MCT detector, coupled with a Bruker Vertex 70 interferometer. The aperture was typically set to $15 \times 15 \mu \mathrm{m}$ and spectra recorded using 512 scans, $8 \mathrm{~cm}^{-1}$ resolution.

For comparison, the slides that had previously been analysed at Daresbury and Elettra were re-analysed using a Varian FTS 7000 spectrometer coupled to a UMA 600 microscope and an MCT detector. An aperture of approximately $100 \times 100 \mu \mathrm{m}$ (approximately 50 cells) was used and spectra recorded using 256 scans at $4 \mathrm{~cm}^{-1}$ resolution. 


\subsection{Data processing}

All spectra were converted to comma separated values files (csv) using the Omnic (Thermo Scientific) software prior to importing into Matlab (Mathworks, Natick, MA, USA). Spectra displaying poor signal to noise and or significant absorptions due to water vapour and $\mathrm{CO}_{2}$ were rejected. The remaining spectra were corrected using the Resonant Mie Scattering (RMieS) correction

Table 1 Cell distribution determined by flow cytometry

\begin{tabular}{llll}
\hline $\mathrm{IC}_{50} 24 \mathrm{~h}$ & $\mathrm{G} 1(\%)$ & $\mathrm{S}(\%)$ & $\mathrm{G} 2 \mathrm{M} \mathrm{( \% )}$ \\
\hline Control & 56 & 26 & 18 \\
KF0101 & 43 & 31 & 26 \\
Cisplatin & 53 & 7 & 40 \\
Methotrexate & 65 & 18 & 17 \\
5FU & 70 & 6 & 24 \\
Paclitaxel & 5 & 4 & 91 \\
\hline
\end{tabular}

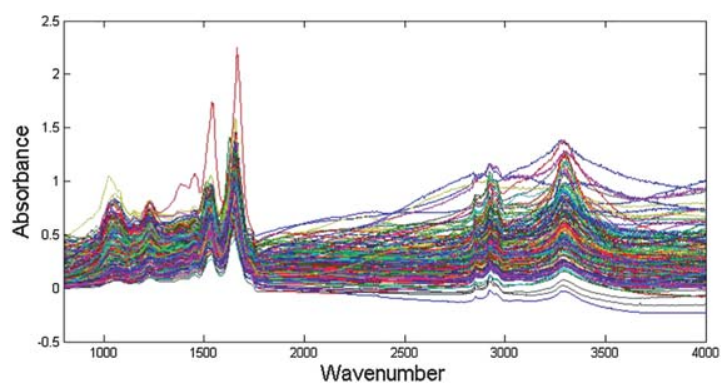

(a)

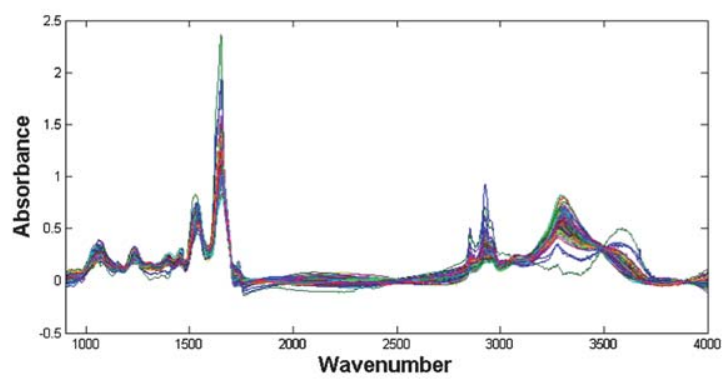

(b)

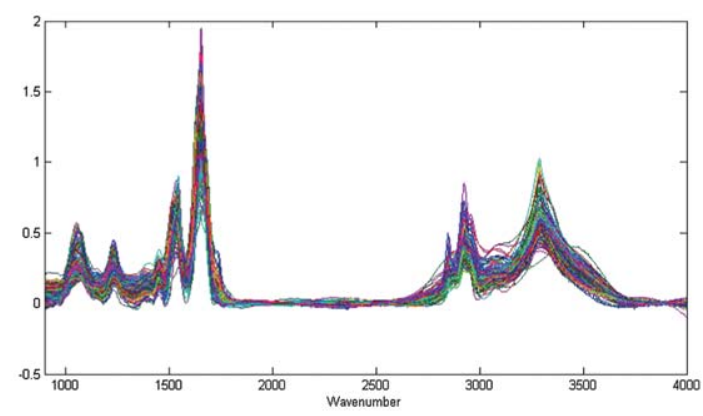

(c)

Fig. 2 SR-IRMS spectra of singles cells of the untreated A2780 cell line (a) Raw spectra; (b) after 1 iteration of RMieS; (c) after 8 iterations of RMieS. algorithm. ${ }^{24}$ This algorithm uses an iterative procedure to improve the correction and the data presented here are the result of eight iterations. The corrected spectra were converted to first derivative, the spectral range abridged to $900-1800 \mathrm{~cm}^{-1}$ prior to vector normalisation and mean centring. The fingerprint region $900-1400 \mathrm{~cm}^{-1}$ was then analysed using PCA analysis (Matlab).

\section{Results and discussion}

\subsection{Flow cytometry}

Table 1 shows the cell-cycle distribution of the A2780 cells studied in this investigation determined by flow cytometry. It is evident that treatment with different drugs affects the cell distribution within the cell cycle. This data indicates that if biochemical changes can be observed using SR-IRMS then separation of the drug treated cells from the control and each other using chemometric methods should be possible. There has to be one caveat to this hyothesis: This investigation is looking at single cells which remain ovarian A2780 cells even after treatment with a drug and are likely to exhibit characteristics similar to the control and so the drug treated cells may well be sub-sets of the control (vide infra).

\subsection{SR-IRMS}

Fig. 2 (a) shows SR-FTIR spectra of individual cells of the A2780 cell line that have not been treated with any drug and represent

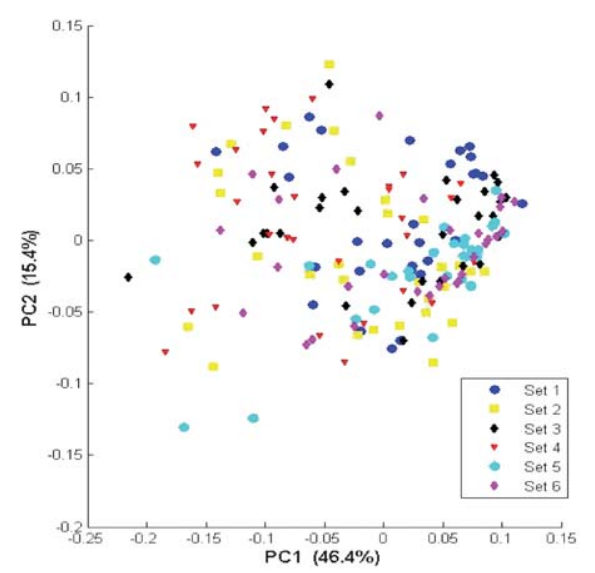

(a)

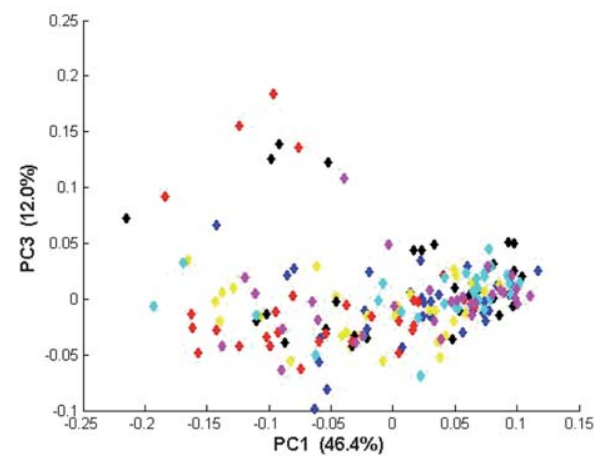

(b)

Fig. 3 PCA analysis of control spectra: (a) PC1 vs. PC2, (b) PC1 vs. PC3. 


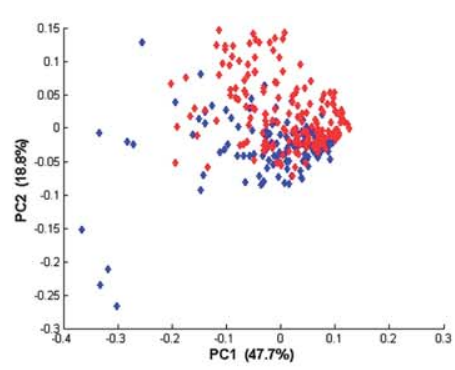

(i)

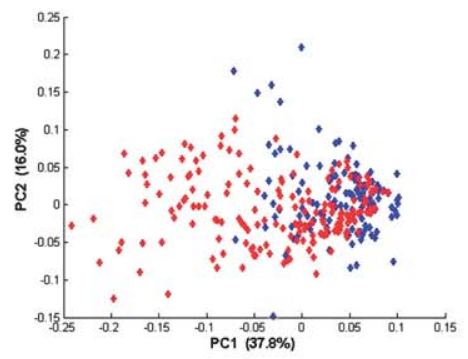

(ii)

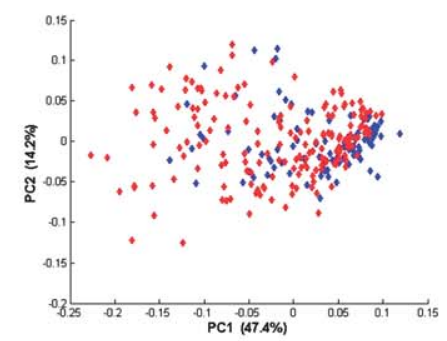

(iii)

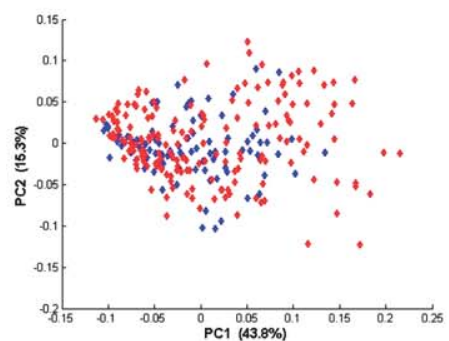

(iv)

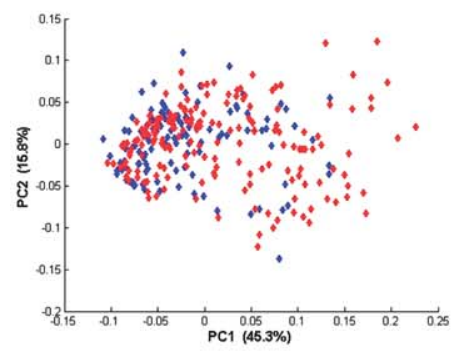

(v)

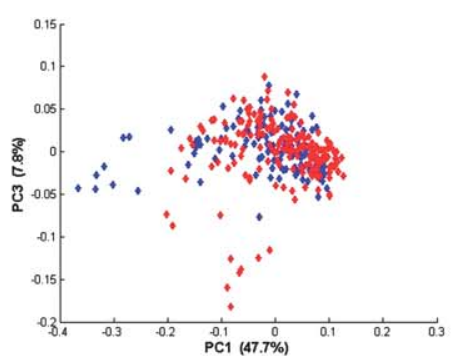

(i)

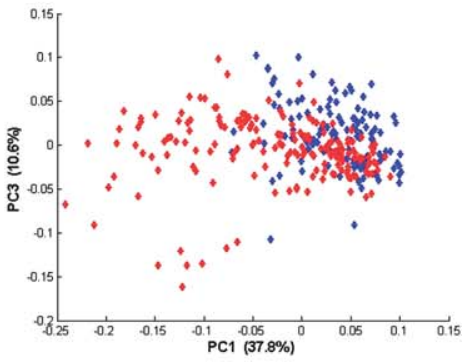

(ii)

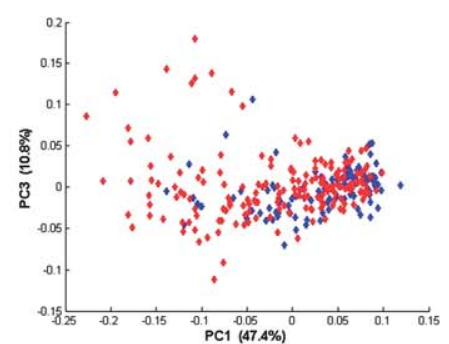

(iii)

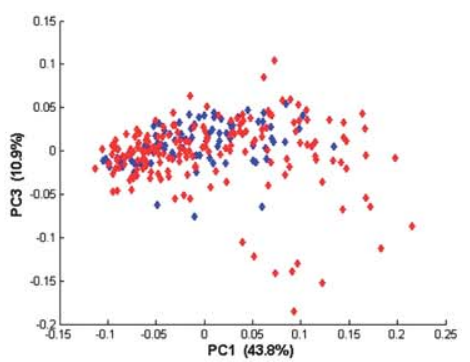

(iv)

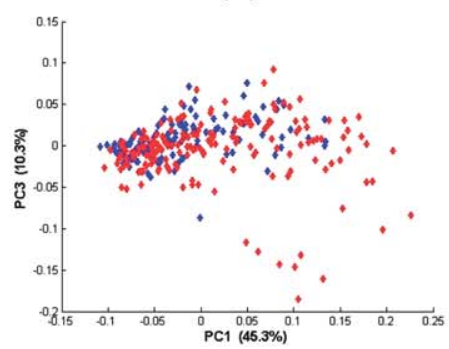

(v)

(A)

(B)

Fig. 4 PCA scores plots of the control cell spectra (red dots) and drug treated cell spectra (blue dots); (A) PC1 vs. PC2, (B) PC1 vs. PC3, (i) KF0101; (ii) cisplatin; (iii) methotrexate; (iv) 5-FU; (v) taxol.

the control data. It is well documented that RMieS is a serious problem when interpreting single cell infrared spectra ${ }^{23}$ and these physical effects are clearly visible in these spectra.
To correct for these effects the RMieS algorithm was used through eight iterations. ${ }^{24}$ Considering the control data, it can be seen from Fig. 2 (b) that a single iteration removes the majority 


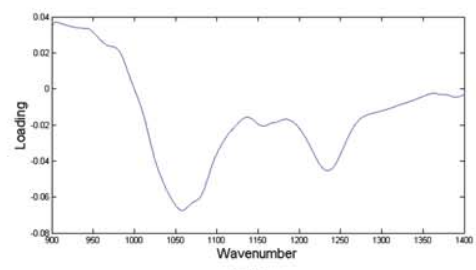

PC1

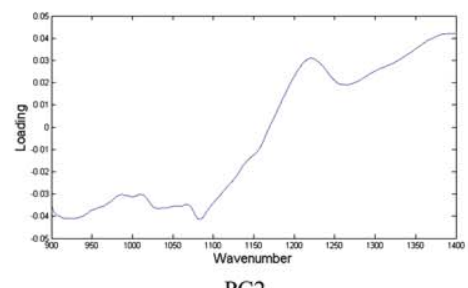

PC2

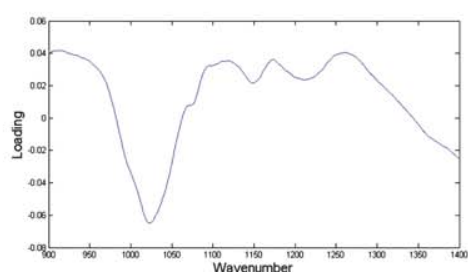

PC3

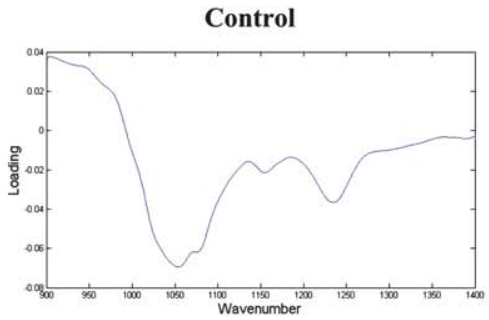

PC1

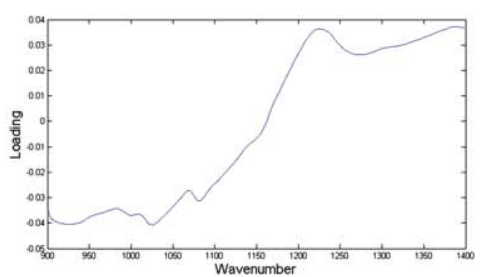

PC2

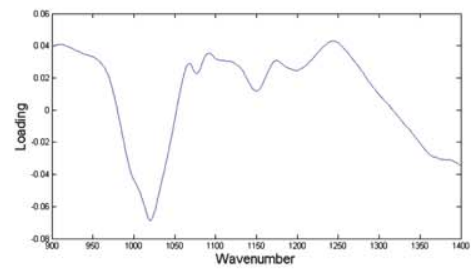

PC3

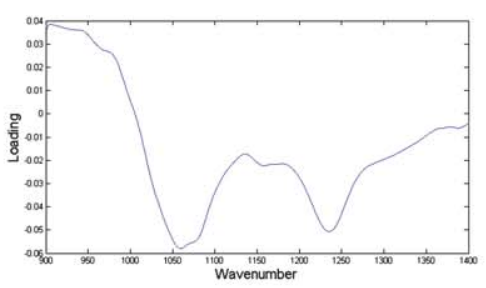

PC1

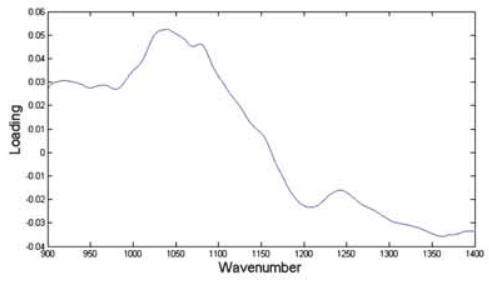

PC2

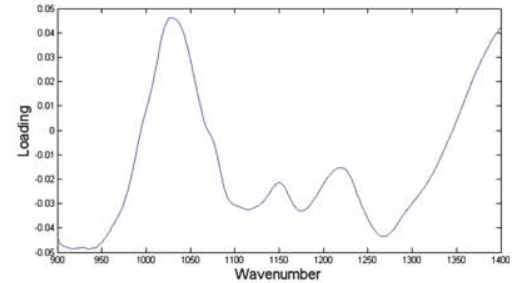

PC3

Cisplatin v control.

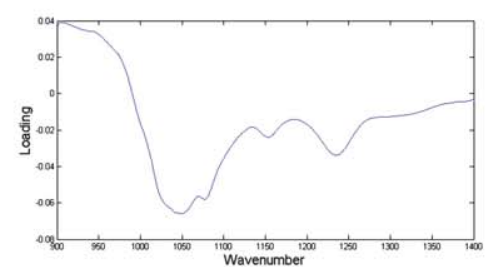

PC1

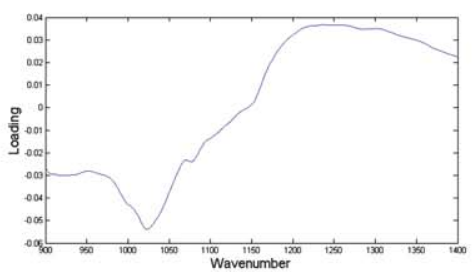

PC2

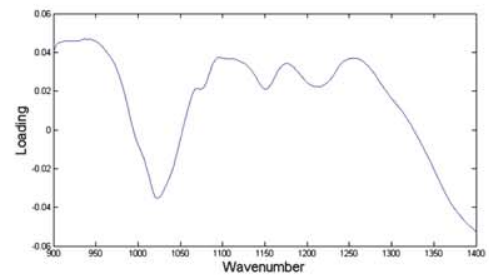

PC3

KF0101 versus cisplatin

KF0101 versus control

Fig. 5 Loading plots for control, control vs. KF0101, control vs. cisplatin and KF0101 vs. cisplatin.

of the scattering effects and after eight iterations Fig. 2 (c) essentially no scattering effects are discernable. The same is true for all other spectral sets collected, see ESI. $\%$
The control data were collected in six blocks of 30 spectra and after correction were subjected to PCA together in the fingerprint region $900-1400 \mathrm{~cm}^{-1}$. The data were analysed in this region as it 
was found that when analysing drug treated cells the greatest spectral changes were observed in this region (vide infra). Each individual set is represented by a different colour in Fig. 3. It is evident that the control spectra are quite heterogeneous and there is also heterogeneity between sets. Only the yellow data set (set 2) spans the entire area defined by the control with other sets being more concentrated into sub-areas. These data suggest that due to the heterogeneity of the spectral characteristics of a single cell population and since not every phase of the cycle is equally populated (Table 1) a large number of data points are required to adequately define the control. This observation is also consistent with the flow cytometry data Table 1 . Furthermore, the heterogeneity of cell populations has been described previously by Diem et al. using bench top IRMS. ${ }^{18,33}$ In their studies they were able to clearly differentiate between cells in the G1 and G2 phases, but found that cells in the $\mathrm{S}$ phase were much more heterogeneous and when combined with G1 and G2 spectra it was difficult to maintain separation and a heterogeneous was spread observed. The data presented here are consistent with that observation.

SR-FTIR spectra of the single A2780 cells treated with one of the drugs, KF0101, cisplatin, methotrexate, 5-FU and taxol (paclitaxel) were initially compared to the control, and the resulting PCA scores plots are shown in Fig. 4. The most striking features in all cases are: (i) The treated cells are essentially defined by specific areas of the control; and (ii) Very few of the treated cells lie outside of the area defined by the control. This indicates that the differences in the spectra are not due to the presence of spectral features of the drug. The spectral characteristics of each drug are significantly different from each other in the region analysed and if separation purely due to drug were observed separate clustering should be expected for treated cells and the control along PC1. The analysis suggests otherwise and the impact of the drug is being observed through biochemical changes that manifest themselves in the IR spectra.

This becomes evident on inspection of the loading plots for the control, control vs. cisplatin, control vs. KF0101, and KF0101 vs. cisplatin, Fig. 5. The bands responsible for the differences in the principal components are shown in Table 2.

The bands responsible for the separation of the control cells along $\mathrm{PC} 1$ can be assigned ${ }^{45}$ to changes in deoxyribose $\left(1057 \mathrm{~cm}^{-1}\right)$, phosphate $\left(1079 \mathrm{~cm}^{-1}\right), \mathrm{C}-\mathrm{O}$ in protein and carbohydrate $\left(1155 \mathrm{~cm}^{-1}\right)$, amide III and nucleic acid phosphate $\left(1234 \mathrm{~cm}^{-1}\right)$ which are all negative and a broad positive region at $950 \pm 50 \mathrm{~cm}^{-1}$ which is difficult to assign with any certainty. Similar changes are seen in $\mathrm{PC} 1$ when the control is compared to both cisplatin and KF0101. Since both cisplatin and KF0101 treated cells appear to be subsets of the control the implication is that in these cases PC1 represents separation based upon where the cell is in the cell cycle. PC1 shows almost the same band pattern when KF0101 and cisplatin are compared save the most intense negative band is at $1046 \mathrm{~cm}^{-1}$ indicative of changes in glycogen. ${ }^{45}$ Comparison of PC2 plots for cisplatin and KF0101 $v s$. the control and the control show the most significant differences are in cisplatin $v s$. control, a clear observation of cell cycleplus behaviour. There is a significant increase in bands assigned to ribose $\left(966,1039 \mathrm{~cm}^{-1}\right)$ and phosphate $\left(1079 \mathrm{~cm}^{-1}\right)$ and decrease in intensity polysaccharides $\left(1207 \mathrm{~cm}^{-1}\right)$, amide III (1307); whereas in PC2 comparing KF0101 vs. control with the control, the differences from those that can be assigned purely to the cell cycle are less obvious. The most significant positive peaks are at phosphate $\left(1227 \mathrm{~cm}^{-1}\right)$ and amide (III) $\left(1305 \mathrm{~cm}^{-1}\right)$, with the most intense negative peaks at ribose $\left(998 \mathrm{~cm}^{-1}\right)$ glycogen $\left(1026 \mathrm{~cm}^{-1}\right)$ and phosphate $\left(1081 \mathrm{~cm}^{-1}\right)$. Comparing KF0101 and cisplatin on PC2 negative peaks for ribose $\left(998 \mathrm{~cm}^{-1}\right)$, glycogen $\left(1023 \mathrm{~cm}^{-1}\right)$ and phosphate $\left(1079 \mathrm{~cm}^{-1}\right)$ and positive peaks for the amide III $\left(1240\right.$ and $\left.1301 \mathrm{~cm}^{-1}\right)$. The same observations are true in the respective PC3 plots: KF0101 is most similar to the control and cisplatin shows clear cell cycle-plus behaviour. For cisplatin a clear increase in glycogen $\left(1028 \mathrm{~cm}^{-1}\right)$ with a significant decrease in non-hydrogen bonded $\mathrm{CH}-\mathrm{OH}\left(1175 \mathrm{~cm}^{-1}\right)$ and phosphate $\left(1268 \mathrm{~cm}^{-1}\right)$, whereas the reverse is seen for KF0101 treated cells and is most comparable to that seen for the control.

It is known that cisplatin, methotrexate, 5-fluorouracil and paclitaxel operate by different mechanisms and at different parts of the cell cycle. ${ }^{37-41}$ The similarity of but subtly different nature of the clustering of the cells treated with these drugs and the clustering along PC1, which essentially represents the cell cycle, fits with this fact, Fig. 4. An intriguing observation from these data is there are a number of spectra that lie outside of the region defined by the control. Thus, in addition to the drug inducing a normal cell cycle response, a response out-side of the normal control cell cycle may well be evident and again gives evidence for cell cycle-plus behaviour. This is most evident for the KF0101 spectra, Fig. 4a(i) and b(i). There are several possible reasons for this: (i) The control area is not completely defined (see earlier

Table 2 Bands responsible for each principal component

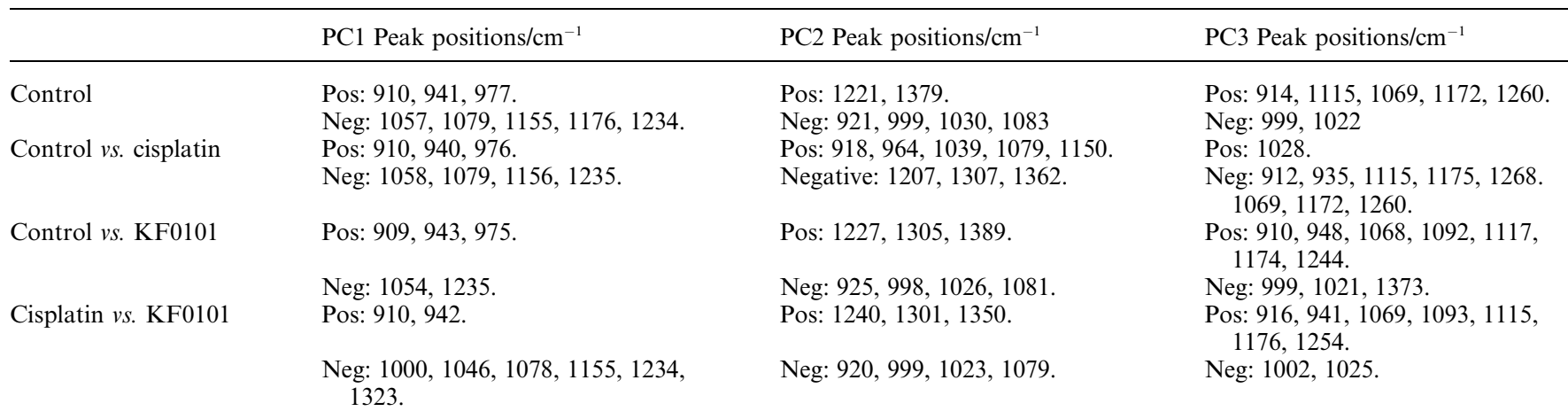


discussion); (ii) It is known that the A2780 cell line develops resistance and inherently resistant cells may respond in an abnormal biochemical way on treatment with the drugs and so appear outside of the area defined by the control and (iii) The drugs may induce necrosis rather than apoptosis and these biochemical properties may not be adequately defined by the control that was used.
Comparison of KF0101 with each of the individual drugs is shown in Fig. 6, and illustrates again that the effect of the drugs on the cell biochemistry can be separated using PCA.

Similar scatter patterns are seen when KF0101 is compared with two different drugs, for example cisplatin and 5FU, Fig. 7. Once again it is evident that the cells treated with different drugs separate from each other on the basis of different biochemistry
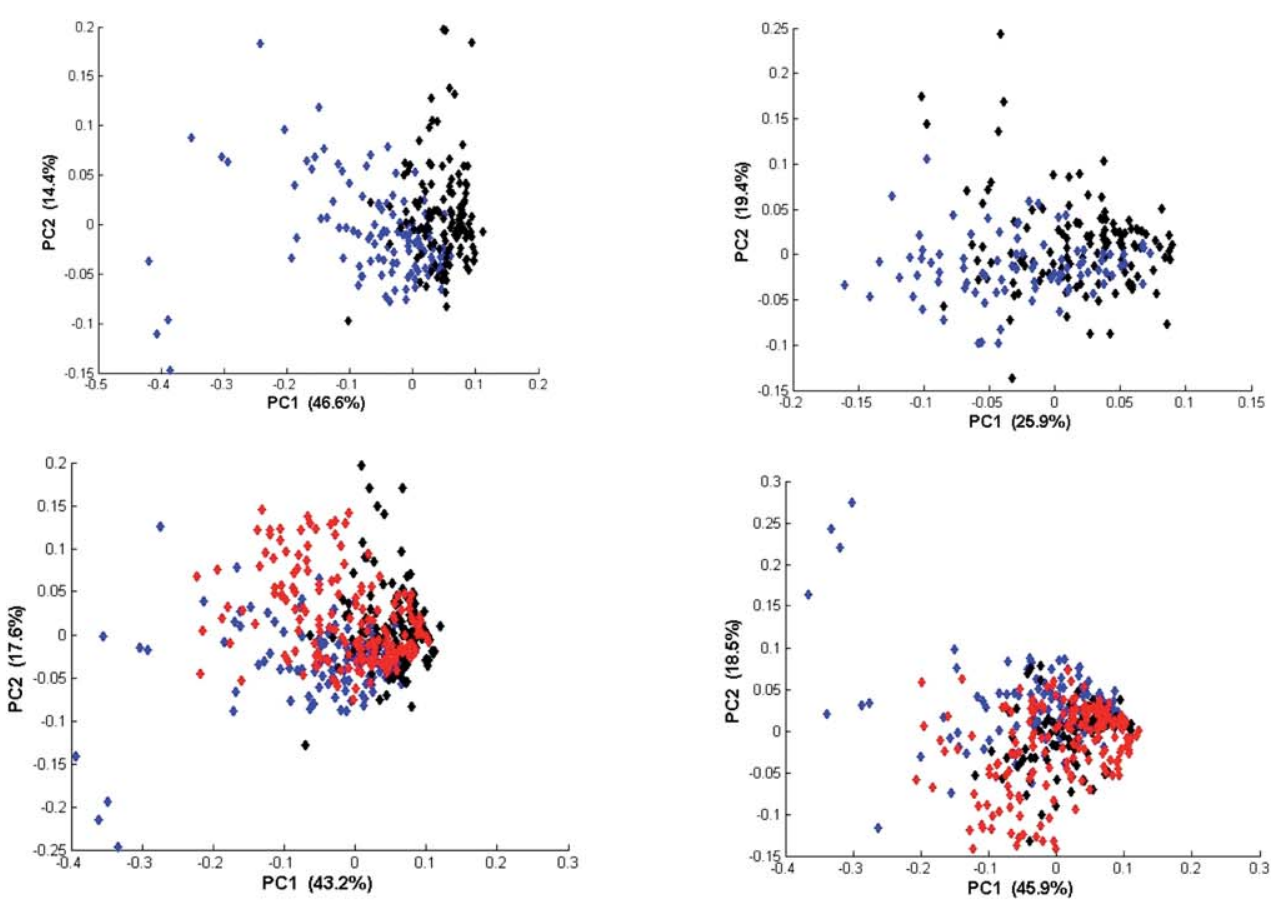

KF0101 vs cisplatin
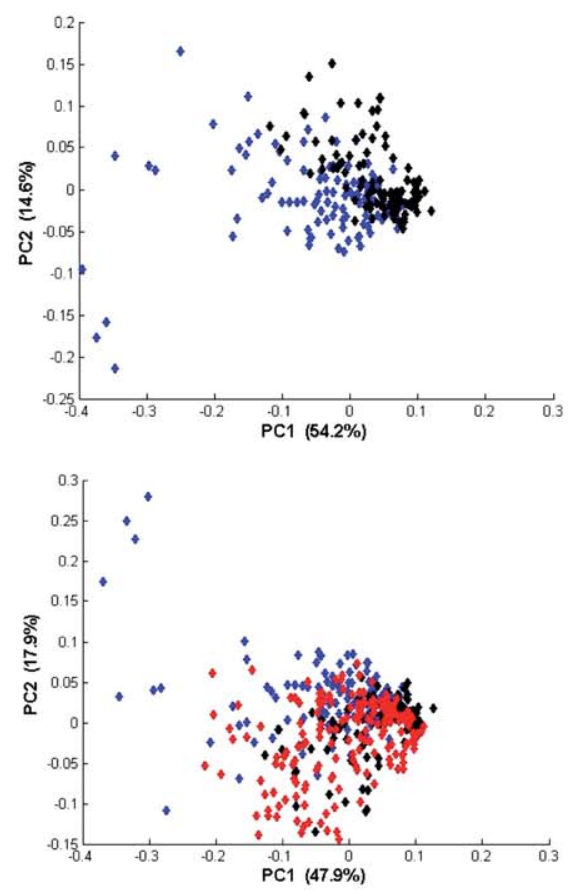

KF0101 versus methotrexate

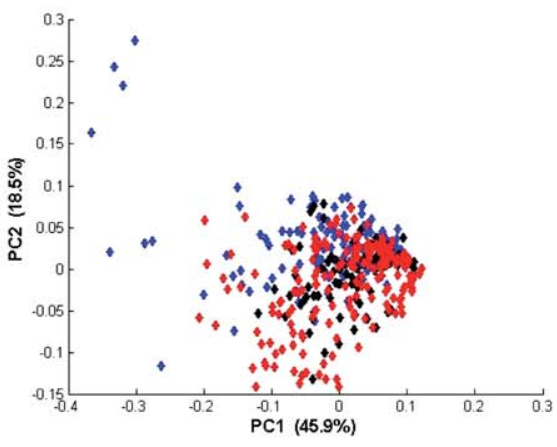

KF0101 versus 5FU
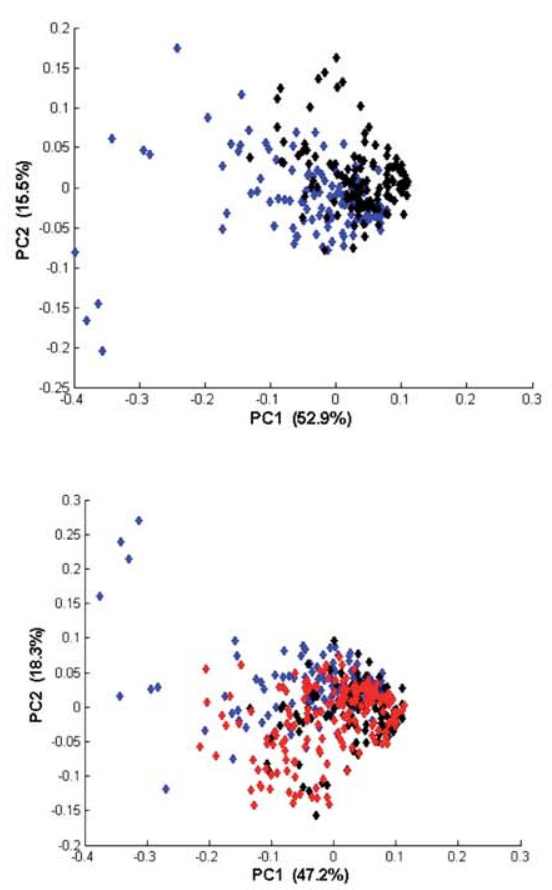

KF0101 vs taxol

Fig. 6 PCA plots of KF0101 (blue) other black and control red. 
and are generally confined to specific parts of the control PCA space indicating drug treatment affects the normal cell cycle processes.

When all of the drugs are combined in one analysis the same general observations are made, Fig. 8.

\subsection{Benchtop IRMS}

The same cell samples have been analysed using benchtop-IRMS with a $100 \times 100 \mu$ aperture (approx 50 cells) and the data collected shows a clear sparation between the control and cells treated with KF0101 and cisplatin, Fig. 9. So, is this consistent with the SR-IRMS data?

The working hypothesis that cells in a different part of the cell cycle give rise to a different spectral profile is key to the success of this and related studies. One reason that a clear separation is observed here is that the benchtop IRMS data looks at the average spectrum of the cell poulation rather than at the spectrum for an individual cell and this can be shown in the following way. Considering the data presented in Table 1 (flow cytometry) the average control spectrum in this study should be made up of the following spectral contributions: $56 \% \mathrm{G} 1,26 \% \mathrm{~S}$, and $18 \%$ G2M; with that for KF0101: 43\% G1, 31\% S, and 26\% G2M; and cisplatin: $53 \% \mathrm{G} 1,7 \% \mathrm{~S}$, and $40 \% \mathrm{G} 2 \mathrm{M}$ respectively. The average cell spectrum for each group should therefore be different and separable using chemometric methods. To test this view we randomly selected 50 spectra from each data set (control, KF0101 and cisplatin) and averaged each set of 50 spectra to generate a single average spectrum for each data set. This process was repeated 20 times for each data set. This generated 60 average spectra from the single cell data. These were then subjected to the same PCA analysis as the single cell data. This
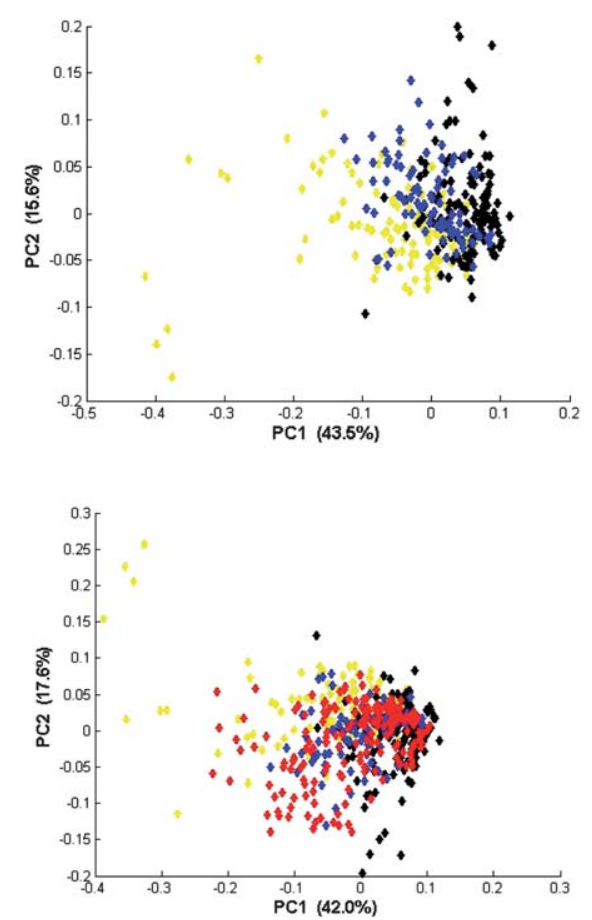

Fig. 7 KF0101 (yellow) vs. cisplatin (black) vs. 5-FU (blue) vs. control (red).
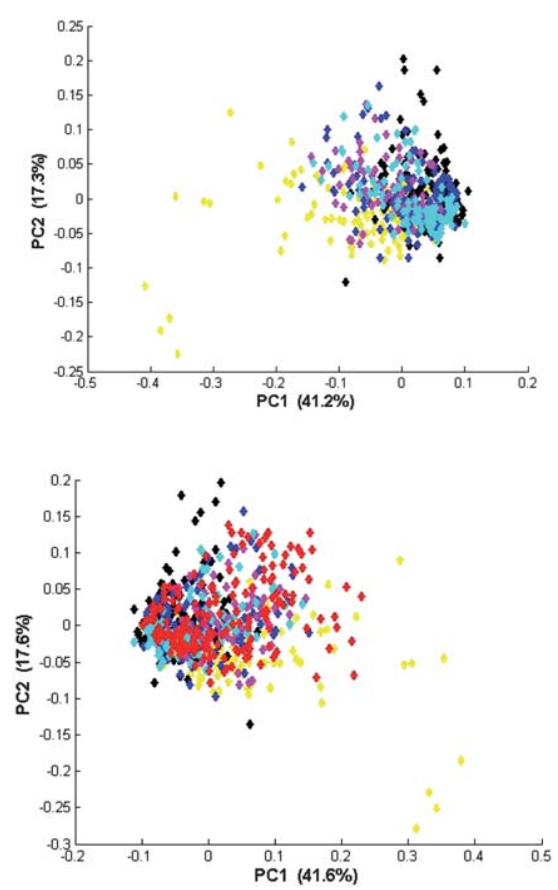

Fig. 8 All drugs combined with and without the control, KF0101 (yellow); Cisplatin (black); methotrexate (cyan); taxol (blue); 5-FU (magenta); control (red).

process was repeated three times and the same result was obtained on each occasion. Clear separation of both cisplation and KF0101 from the control is observed affording a result that is consistent with that obtained by benchtop-IRMS, Fig. 10.

It is clear therefore that benchtop IRMS gives an average spectrum derived from the cell population made up from a collection of single cells at different parts of the cell cycle, whereas single cell spectra are representative of where the cell is in the cell cycle. If single cell spectra are averaged and then compared the data is analogous to that obtained in multicellular work. This can be seen by comparing Fig. 3 (a) with Fig. 6 (ii). From the distribution seen in Fig. 6 (ii) the average spectrum defined by the control is going to be different from that defined by KF0101 and or cisplatin. One should therefore expect tighter more well defined clusters in multicellular work when compared to single cell SR-IRMS data as the heterogeneity of the single cell spectra is averaged. The SR-IRMS data also confirms that the

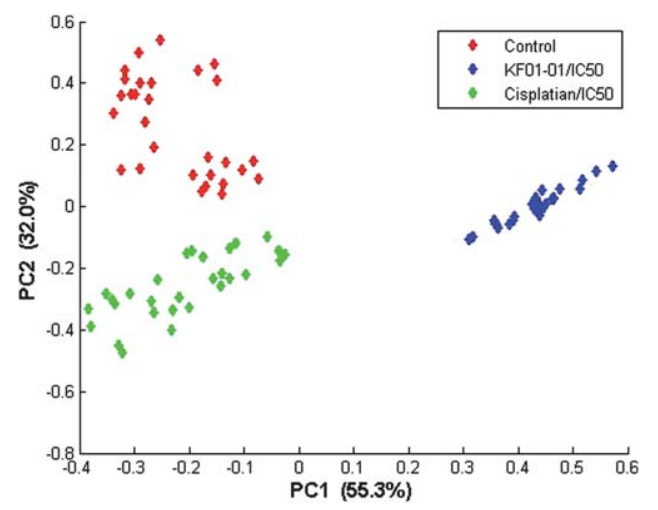

Fig. 9 PCA plot of KF0101 vs. cisplatin vs. control. 


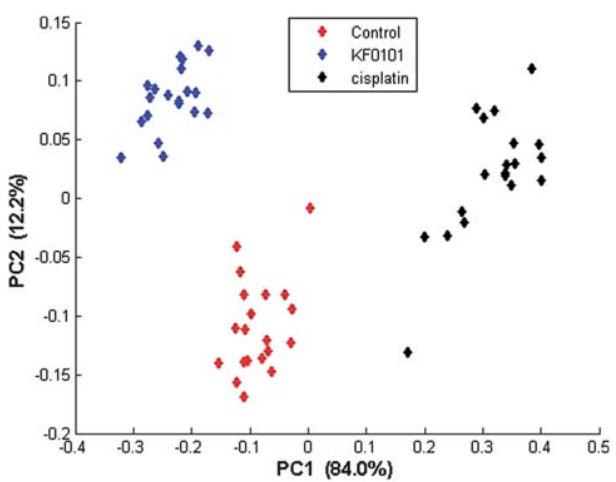

Fig. 10 PCA scores plot of averaged single cell spectra of control $v$. KF0101 vs. cisplatin.

separation seen on the multicellular work is not just due to differences in drug spectra. It is now clear that using SR-IRMS to study single cells offers an opportunity to gain greater insight into what is going on at the cellular level when compared to multicellular work, where subtle differences between similarly treated cells are averaged. For example, PC1 in this SR-IRMS study is shown to be essentially indicating where the cell sits relative to the normal cell cycle and that differences in other principal components are indicative of drug induced cell cycleplus behaviour. SR-IRMS studies therefore appear able to give information on cell cycle perturbations relating to drug treatment that may not be evident in multicellular work due to spectral averaging.

\section{Conclusions}

In this study it has been shown that A2780 ovarian cells when treated with a range of different drugs known to operate by different mechanisms can be distinguished from each other and untreated A2780 cells using SR-IRMS. The observed heterogeneity of the control spectra is due to cells being at different points in the cell cycle and the differences in IR spectra exhibited by cells at different points of the cycle. Therefore large number of single cell control spectra are required to adequately define the control and enable conclusions to be drawn about the impact of drug treatment. The majority of spectra obtained from the drug treated samples when analysed by PCA fall into certain areas of the control PCA space, with the areas of concentration subtly different for each drug which can be linked to how and where in the cell cycle the drug of choice acts. The data is also consistent with that obtained from benchtop IRMS, shown through averaging of the single cell data. This study clearly shows that single cell studies using SR-IRMS affords more detail about changing cell biochemistry than can be obtained from multicellular work. PC1 is essentially the same in all comparisons and is indicative of where the cell sits in the cell cycle. More subtle differences from the control are observed in higher principal components and these contain information on the impact of the drug on the cell's biochemistry: cell cycle-plus behaviour. A number of drugtreated cell spectra are observed outside of the area defined by the normal cell cycle. This further indicates that there may be additional cell cycle-plus information to be obtained.

\section{Acknowledgements}

This work has been supported through the following awards: Daresbury SRS: 49243 and 50169; Elettra 20085256, 20090081, 20095373, and the EU is acknowledged for travel to Elettra under the ELISA (226716) scheme. PJM thanks the EPSRC for a studentship and KRF thanks Modern Biosciences for financial support. The EPSRC/RSC Analtical Science PhD Studentship Scheme is acknowledged for support for PB and EJ.

\section{Notes and references}

1 P. T. T. Wong, R. K. Wong, T. A. Caputo, T. Goodwin and B. Rigas, IR spectroscopy of exfoliated human cervical cells; evidence of extensive structural changes during carcinogenesis, Proc. Natl. Acad. Sci. U. S. A., 1991, 88, 10988-10992.

2 M. Diem, M. Romeo, S. Boydston-White, M. Miljković and C. Matthäus, A decade of vibrational micro-spectroscopy of human cells and tissue (1994-2004), Analyst, 2004, 129, 880-885.

3 D. I. Ellis and R. Goodacre, Metabolic fingerprinting in disease diagnosis: biomedical applications of infrared and Raman spectroscopy, Analyst, 2006, 131, 875-885.

4 M. Diem, S. Boydston-White and L. Chiriboga, Infrared Spectroscopy of Cells and Tissues: Shining Light onto a Novel Subject, Appl. Spectrosc., 1999, 53, 148A-161A.

5 C. Kendall, M. Isabelle, F. Bazant-Hegmark, J. Hutchings, L. Orr, J. Babarah, R. Baker and N. Stone, Vibrational spectroscopy:a clinical tool for cancer diagnostics, Analyst, 2009, 134, 1029-1045.

6 G. Birarda, G. Grenci, L. Businaro, B. Marmiroli, S. Pacor, F. Piccirilli and L. Vaccari, Infrared microspectroscopy of biochemical response of living cells in microfabricated devices, Vib. Spectrosc., 2010, 53, 6-11.

7 J. Pijanka, G. D. Sockalingum, A. Kohler, Y. Yang, F. Draux, G. Parkes, K.-P. Lam, D. Collins, P. Dumas, C. Sandt, D. G. van Pittius, G. Douce, M. Manfait, V. Utereiner and J. Sulé-Suso, Synchrotron-based FTIR spectra of stained single cells. Towards a clinical application in pathology, Lab. Invest., 2010, 90, 797-807.

8 F. Draux, P. Jeanesson, C. Gobinet, J. Sulé-Suso, J. Pijanka, C. Sandt, P. Dumas, M. Manfait and G. D. Sockalingum, IR spectroscopy reveals effect of non-cytotoxic doses of anti-tumour drug on cancer cells, Anal. Bioanal. Chem., 2009, 395, 2293-2301.

9 K. L. Goff, L. Quaroni and K. E. Wilson, Measurement of metabolite formation in single living cells of Chlamydomonas reinhardtii using synchrotron Fouriour-Transform Infrared spectromicroscopy, Analyst, 134, pp. 2216-2219.

10 J. K. Pijanka, A. Kohler, Y. Yang, P. Dumas, S. Chio-Srichan, M. Manfait, G. D. Sockalingum and J. Sulé-Suso, Spectroscopic signatures of single, isolated cancer cell nuclei using synchrotron infrared microscopy, Analyst, 2009, 134, 1176-1181.

11 N. Kaun, S. Kaulka, J. Frank, U. Schade, M. J. Vellekoop, M. Harasek and B. Lendl, Towards biochemical reaction monitoring using FT-IR synchrotron radiation, Analyst, 2006, 131, 489-494.

12 E. Gazi, J. Dwyer, N. P. Lockyer, J. Miyan, P. Gardner, C. A. Hart, M. D. Brown and N. W. Clarke, A study of cytokinetic and motile prostate cancer cells using synchrotron-based FTIR micropectroscopic imaging, Vib. Spectrosc., 2005, 38, 193-201.

13 E. Gazi, J. Dwyer, N. P. Lockyer, J. Miyan, P. Gardner, C. Hart, M. Brown and N. W. Clarke, Fixation protocols for subcellular imaging by synchrotron-based Fourier transform infrared microspectroscopy, Biopolymers, 2004, 77, 18-30.

14 N. Jamin, L. Miller, J. Moncuit, W. H. Fridman, P. Dumas and J. L. Teillaud, Chemical Heterogeneity in Cell Death: Combined Synchrotron IR and Fluorescence Microscopy Studies of Single Apoptotic and Necrotic Cells, Biopolymers, 2003, 72, 366-373.

15 H. N. Holman, M. C. Martin, E. A. Blakely, K. Bjornstad and W. McKinney, IR Spectroscopic Characteristics of Cell Cycle and Cell Death Probed by Synchrotron Radiation Based Fourier Transform IR Spectromicroscopy, Biopolymers, 2000, 47, 329-335.

16 N. Jamin, P. Dumas, J. Moncuit, W. H. Fridman, J. L. Teillaud, G. L. Carr and G. P. Williams, Chemical imaging of nucleic acids, proteins, and lipids of a single living cell. Application of 
synchrotron IR microspectroscopy in cell biology, Cell. Mol. Biol., 1998, 44, 9-11.

17 B. Mohlenhoff, M. Romeo, B. R. Wood and M. Diem, Mie-scattering and non-Beer-Lambert absorption behaviour of human cells in infrared microspectroscopy, Biophys. J., 2005, 88, 3635-3640.

18 M. Romeo, B. Mohlenhoff and M. Diem, Infrared microspectroscopy of human cells: Causes for the spectra variance of oral mucosa (buccal) cells, Vib. Spectrosc., 2006, 42, 9-14.

19 M. Romeo and M. Diem, Correction of dispersive line shape artefact observed in diffuse reflection infrared spectroscopy and absorbtion/ reflection (transflection) infrared micro-spectroscopy, Vib. Spectrosc., 2005, 38, 129-132.

20 J. Lee, E. Gazi, J. Dwyer, M. D. Brown, N. W. Clarke, J. M. Nicholson, and P. Gardner, Optical artefacts in transflection mode FTIR microspectroscopic images of single cells ona biological support: the effect of back-scattering into collection optics, 2007, 132, pp. $750-755$.

21 A. Kohler, J. Sulé-Suso and G. D. Sockalingum, Estimating and correcting Mie scattering in synchrotron-based microspectroscopic FTIR spectra by extended multiplicative signal correction (EMSC), Appl. Spectrosc., 2008, 62, 259-266.

22 P. Bassan, H. J. Byrne, J. Lee, F. Bonnier, C. Clarke, P. Dumas, E. Gazi, M. D. Brown, N. W. Clarke and P. Gardner, Reflection contributions to dispersion artefact in FTIR spectra of cellular samples, Analyst, 2009, 134, 1171-1175.

23 P. Bassan, H. J. Byrne, F. Bonnier, J. Lee, P. Dumas and P. Gardner, Resonant Mie scattering in infrared spectroscopy of biological materials - understanding the 'dispersion artefact', Analyst, 2009, 134, 1586-1593.

24 P. Bassan, A. Kohler, H. Martens, J. Lee, H. J. Byrne, P. Dumas, E. Gazi, M. Brown, N. Clarke and P. Gardner, Resonant Mie Scattering (RMieS) correction of infrared spectra from highly scattering biological samples, Analyst, 2010, 135, 268-277.

25 P. Bassan, A. Kohler, H. Martens, J. Lee, E. Jackson, N. Lockyer, P. Dumas, M. Brown, N. Clarke, P. Gardner, RMieS-EMSC correction for infrared spectra of biological cells: Extension using full Mie theory and GPU computing, J. Biophotonics, DOI: $10.1002 /$ jbio. 201000036 .

26 R. Gasper, J. Dwelle, R. Kiss, T. Mijatovic and E. Goormaghtigh, IR Spectroscopy as a new tool for evidencing antitumor drug signatures, Biochim. Biophys. Acta, 2009, 1788, 1263, 1270.

27 T. J. Harvey, A. Henderson, E. Gazi, N. W. Clarke, M. D. Brown, E. Correia Faria, R. D. Snook and P. Gardner, Discriminationof prostate cancer cells by reflection mode FTIR photoacustic spectroscopy, Analyst, 2007, 132, 292-295.

28 E. Gazi, M. Baker, J. Dwyer, N. P. Lockyer, P. Gardner, J. H. Shanks, R. S. Reeve, C. A. Hart, N. W. Clarke and M. D. Brown, A Correlation of FTIR Spectra Derived from Prostate Cancer Biopsies with Gleason Grade and Tumour Stage, Urology, 2006, 50, 750-761.

29 E. Gazi, N. P. Lockyer, J. C. Vickerman, P. Gardner, J. Dwyer, C. A. Hart, M. D. Brown, N. W. Clarke and J. Miyan, Imaging ToF-SIMS and synchrotron-based FT-IR microspectroscopic studies of prostate cancer cell lines, Appl. Surf. Sci., 2004, 231-232, $452-456$.

30 E. Gazi, J. Dwyer, N. P. Lockyer, P. Gardner, J. C. Vickerman, J. Miyan, C. A. Hart, M. Brown, J. H. Shanks and N. Clarke, The combined application of FTIR microspectroscopy and ToF-SIMS imaging in the study of prostate cancer, Faraday Discuss., 2004, 126, 41-59.

31 E. Gazi, J. Dwyer, P. Gardner, A. Ghanbari-Siahkali, A. P. Wade, J. Miyan, N. P. Lockyer, J. C. Vickerman, N. W. Clarke, J. H. Shanks, L. J. Scott, C. A. Hart and M. Brown, Applications of Fourier transform infrared microspectroscopy in studies of benign prostate and prostate cancer: A pilot study, J. Pathol., 2003, 201, 99-108.

32 E. Gazi, P. Gardner, N. P. Lockyer, C. A. Hart, M. D. Brown and N. W. Clarke, Direct evidence of lipid translocation between adipocytes and prostate cancer cells with imaging FTIR microspectroscopy, J. Lipid Res., 2007, 48, 1846-1856.

33 S. Boydston-White, M. Romeo, T. Chernenko, A. Regina, M. Miljković and M. Diem, Cell-cycle dependent variations in FTIR micro-spectra of single proliferating HeLa cells: Principal component and artificial neural network analysis, Biochim. Biophys. Acta, 2006, 1758, 908-914.

34 A. Hammiche, M. J. German, R. Hewitt, H. M. Pollock and F. L. Martin, Monitoring Cell Cycle Distribution in MCF-7 Cells Using Near-Field Photothermal Microspectroscopy, Biophys. J., 2005, 88, 3699-3706.

35 J. R. Mourant, Y. R. Yamada, S. G. Carpenter, L. R. Dominique and J. P. Freyer, FTIR Spectroscopy Demonstrates Biochemical Differences in Mammalian Cell Cultures at Different Growth Stages, Biophys. J., 2003, 85, 1938-1947.

36 K. R. Flower, A. T. McGown, P. J. Miles, R. G. Pritchard and J. E. Warren, Isolation of $1,4-\mathrm{Li}_{2} \mathrm{C}_{6} \mathrm{H}_{4}$ and its reaction with $\left[\left(\mathrm{Ph}_{3} \mathrm{P}\right) \mathrm{AuCl}\right]$, Dalton Trans., 2010, 39, 3509-3520.

37 D. G. Johnson and C. L. Walker, Cyclins and Cell Cycle Checkpoints, Annu. Rev. Pharmacol. Toxicol., 1999, 39, 295-312.

38 E. S. L. Chan and B. N. Cronstein, Methotrexate-how does it really work, Nat. Rev. Rheumatol., 2010, 6, 175-178.

39 R. Yoshikawa, M. Kusunoki, H. Yanagi, M. Noda, J. I. Furuyama, T. Yamamura and T. Hashimoto-Tamaoki, Dual antitumor effects of 5-fluorouaracil on the cell cycle in colorectal carcinoma cells: a novel target mechanism concept for pharmacokinetic modulating chemotherapy, Cancer Res., 2001, 61, 1029-1037.

40 T.-Z. Wang, H.-S. Wang and Y.-K. Soong, Paclitaxel- Induced Cell Death, Cancer, 2000, 88, 2619-2628.

41 M. A. Barry, C. A. Behnke and A. Eastman, Activation of programmed cell death (Apoptosis) by cisplatin, other anticancer drugs, toxins and hypothermia, Biochem. Pharmacol., 1990, 10, 2353-2362.

42 M. J. Tobin, M. A. Chesters, J. M. Chalmers, F. J. M. Rutten, S. E. Fisher, I. M. Symonds, A. Hitchcock, R. Allibone and S. Dias-Gunasekara, Infrared microscopy of epithelial cancer cells in whole tissues and in tissue culture, using synchrotron radiation, Faraday Discuss., 2004, 126, 27-38.

43 M. J. Baker, C. Clarke, D. Démoulin, J. M. Nicholson, F. M. Lyng, H. J. Byrne, C. A. Hart, M. D. Brown, N. W. Clarke and P. Gardner, An investigation of the RWPE prostate derived family of cells using FTIR spectroscopy, Analyst, 2010, 135, 887-894.

44 S. Lupi, A. Nucara, A. Perucchi, P. Calvani, M. Ortolani, L. Quaroni and M. Kiskinova, Performance of SISSI, the infrared beamline of the ELETTRA storage ring, J. Opt. Soc. Am., 2007, B24, 959-964.

45 Z. Movasaghi, S. Rehman and I. Rehman, Fouriour Transform Infrared (FTIR) Spectroscopy of Biological Tissues, Appl. Spectrosc. Rev., 2008, 43, 134-179. 